\title{
Comprehensive Testing of a Neon Cryogenic Capillary Pumped Loop
}

\author{
Mark Kobel and Jentung Ku \\ NASA Goddard Space Flight Center, Thermal Engineering Branch, Code 545 Greenbelt, Maryland 20771 \\ (301) 286-8832 Mark.Kobel@gsfc.nasa.gov
}

\begin{abstract}
This paper describes a comprehensive test program of a cryogenic capillary pumped loop (CCPL) using neon as the working fluid in the temperature range between $30 \mathrm{~K}$ and $40 \mathrm{~K}$. The test article was originally designed to be used with nitrogen in the $70 \mathrm{~K}$ to $100 \mathrm{~K}$ temperature range, and was refurbished for testing with neon. Tests performed included start up from a supercritical state, power cycle, sink temperature cycle, heat transport limit, low power limit, reservoir set point change and long duration operation. The neon CCPL has demonstrated excellent performance under various conditions.
\end{abstract}

\section{INTRODUCTION}

A capillary pumped loop (CPL) is a very versatile and robust thermal control device that can transfer a large amount of heat over long distances with very small temperature differences. Room temperature CPLs have been under extensive development over the past two decades and are the baseline design for EOS-Terra and HST Servicing Mission 3. The three primary loops onboard Terra spacecraft have been in full operation for over a year all demonstrate excellent performance. Extending the CPL technology to the cryogenic temperature ranges faces several technical challenges: 1) start-up of the CPL from a supercritical state; 2) coefficient of thermal expansion mismatch between the wick and the evaporator shell; 3) pressure containment of the loop at ambient temperature; and 4) parasitic heat gains along the liquid return line. Through successive development efforts over the past few years, all of these technical issues have been resolved for loops utilizing nitrogen (Baumann, 1998). A cryogenic capillary pumped loop (CCPL) using nitrogen as the working fluid has been flown onboard the Space Shuttle STS-85 in October of 1998, and demonstrated excellent performance between $75 \mathrm{~K}$ and $105 \mathrm{~K}$ for heat loads between $0.5 \mathrm{~W}$ and $12 \mathrm{~W}(\mathrm{Ku}, 1999)$.

Future space missions will require optics at much lower temperatures than $75 \mathrm{~K}$. For example, the NGST will have mirrors operated in the $30 \mathrm{~K}$ to $40 \mathrm{~K}$ temperature range, and future infrared telescope will require mirror temperatures around $3 \mathrm{~K}$. The CCPL operating temperatures are dependent upon available working fluids that meet the thermal and hydraulic requirements. Feasible fluids for temperatures lower than $75 \mathrm{~K}$ include neon (35K), hydrogen (25K) and helium (3K). The next logic step is to develop a neon CCPL in the temperature range of $30 \mathrm{~K}$ to $40 \mathrm{~K}$. A nitrogen CCPL has previously been tested with neon as the working fluid and demonstrated its feasibility (Bugby, 1997). Because of budget and schedule constraints, only a few test runs were conducted. The same CCPL has been refurbished and tested for extensive investigation of its performance characteristics. Tests conducted include start-up from supercritical states, power cycle, sink temperature cycle, heat transport limit, low power limit, reservoir set point change, and long duration operation. Effects of the fluid inventory on the loop operation were also investigated. Details of the test article, test set up and test program are described in the following sections.

\section{Test Article}

The neon CCPL consists of an evaporator, a condenser, a vapor line, a liquid line, a cold and a hot reservoir. A picture of the CCPL, without the hot reservoir is shown in Figure 1. The test article was originally designed and 
tested with nitrogen and has been recently refurbished for testing with neon. The evaporator is $3.8 \mathrm{~cm}$ long with an outer diameter of $1.3 \mathrm{~cm}$ and an active length of $2.5 \mathrm{~cm}$. It is made of $316 \mathrm{~L}$ stainless steel with a 2.5-micron stainless steel wick. The evaporator uses a three-port design with the liquid line plumbed as a bayonet to enhance core flushing during start-up and to increase vapor bubble tolerance. It is swaged into a copper saddle, which provides a $2.5 \mathrm{~cm}$ by $2.5 \mathrm{~cm}$ surface area for sensor mounting. The CCPL was gold plated to minimize parasitic heating.

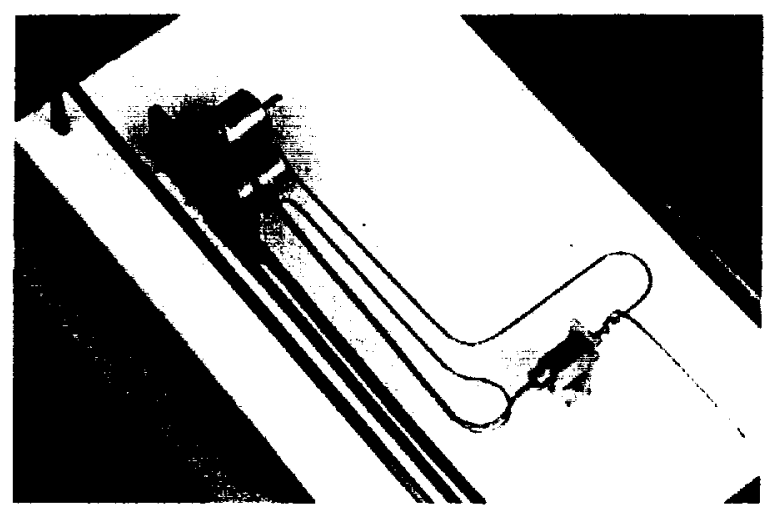

FIGURE 1. Photo of CCPL, without Hot Reservoir.

The vapor line, liquid return line and the cold reservoir feed line are all made of $1.59 \mathrm{~mm}$ outer diameter stainless steel tubing with a $0.15 \mathrm{~mm}$ wall. The condenser design consists of tubing wrapped around a hollow OFHC copper spool which is then fitted to a cryocooler interface. The spool contains 11 grooves per inch on the outer surface to accommodate the condenser and reservoir feed lines. The condenser requires a three-pass design to optimize the integration of the hot reservoir and the liquid cooled shield. See figure 2 for the flow schematic and instrumentation locations. The hot reservoir feed line passes over the condenser spool to chill the fluid, which is displaced from the hot reservoir during the cool down. The hot reservoir line is then plumbed into the midpoint of the condenser. After the reservoir line tee, the condenser line leaves the spool and is used to chill the liquid cooled shield. The condenser line then makes a second pass over the spool before returning to the evaporator. The other leg of the tee makes the third pass over the spool and is the first pass of the vapor line return.

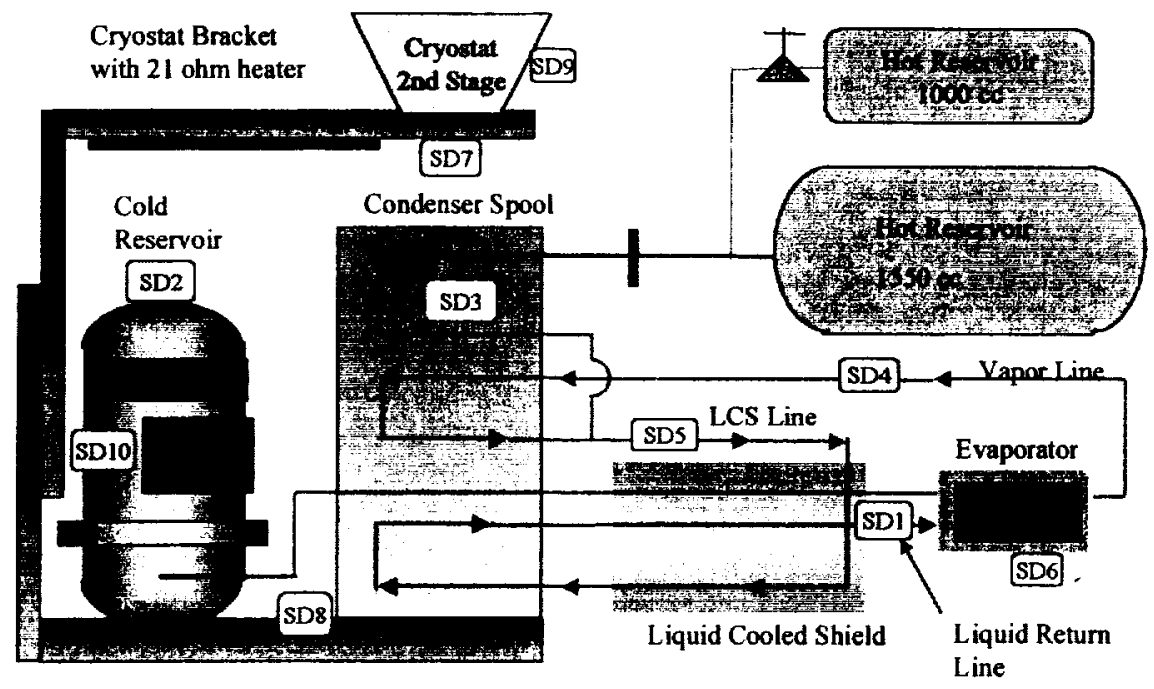

FIGURE 2. Schematic of CCPL.

The cold reservoir is fabricated from $\mathbf{3 1 6}$ stainless steel with flat end caps and an internal polyethylene wick. It is mounted directly to the cooling plate, and is supported by four copper standoffs. The necessary cold biasing is achieved through the conductance of the copper standoffs. The cold reservoir has a total volume of $20.8 \mathrm{cc}$. The hot 
reservoir, originally designed for nitrogen CCPL, is a stainless steel cylinder with an outer diameter of $104.6 \mathrm{~mm}$ and a length of $130 \mathrm{~mm}$ plus two hemispherical end caps to provide a total fluid volume of $1500 \mathrm{cc}$. A secondary hot reservoir with an additional $1000 \mathrm{cc}$ capacity was needed to accommodate the neon working fluid. The hot reservoir is not particularly weight critical, since it is located away from the cryogenic region.

\section{Test Set Up}

The test article was directly mounted to the second stage of the Gifford McMahon cryo-refrigerator inside a cryogenically pumped 18 -inch diameter vacuum chamber. The hot reservoir, absolute pressure transducer and charge manifold were located outside the vacuum chamber. A metering valve was included in the charge manifold to enable controlled charge pressure bleed off. A portable high vacuum pumping station was utilized to evacuate the loop. The charge process included several flush charges prior to the final charge using research grade of neon (99.9995\% purity).

The second stage of the cryogenic refrigerator is capable of dissipating 8.5 watts at $25 \mathrm{~K}$. A dedicated proportional integral derivative (PID) temperature controller maintained the desired temperature of the cryostat second stage (condenser sink). A low temperature interlock for the cryostat prevented accidental freezing of the condenser/reservoir by limiting the minimum temperature of its $2^{\text {nd }}$ stage (neon freezes at $24.5 \mathrm{~K}$ ). A second Lshaped bracket was required to adapt to the original mounting bracket to the cryogenic refrigerator. Both brackets are made of oxygen free high purity copper (OFHC). Grafoil was used as the thermal interface material between all mounting surfaces. Figure 3 shows the CCPL inside the vacuum chamber without multi layer insulation (MLI). A G10 plate mounted to the condenser bracket supported the evaporator. To assure that the evaporator was not in the reflux position, its liquid inlet line was positioned $2 \mathrm{~mm}$ above the highest point of the condenser spool. To test more adverse tilt positions, the OFHC bracket can be rotated at the second stage interface. The entire test set up inside the vacuum chamber was wrapped with 10 layers of MLI, including the cryostat first and second stages. There was no temperature controlled shroud required for this test, only MLI. The chamber environment remained at room temperature.

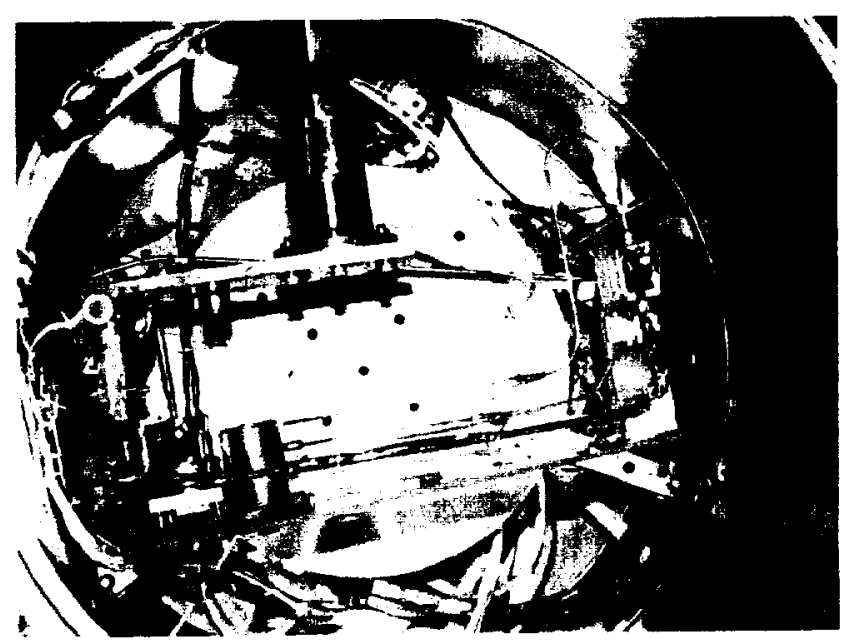

FIGURE 3. CCPL inside Chamber without MLI.

It should be noted that this loop was designed for nitrogen working fluid (90 to 105K working range). Neon's operating range is 35 to $40 \mathrm{~K}$ at the evaporator. No modifications, other than an increased hot reservoir volume were made to accommodate the neon fluid.

\section{Instrumentation}

Special care was taken when applying the instrumentation to the cryocpl. At neon temperature, $30-40 \mathrm{~K}$, parasitics play a larger role. All kapton film heaters and silicone diodes were bonded in place using stycast 2850 epoxy. The locations of the diodes and heaters can be seen in figure 2 . A total of four heaters and 10 silicon diodes were used 
for this test program. The sink temperature (cryostat bracket) and the cold reservoir were controlled to within $0.1 \mathrm{~K}$ with the respective temperature controller during these tests. The evaporator heater power was a four-wire measurement as were all of the silicone diodes. The diode leads included 30 inches of 32-gage phosphor bronze quad lead wire and then joined to 28 gage shielded wire. The heater leads included a 2 inch 32 gage manganin wire and then back to 24 gage copper wire. Both harnesses were individually thermally anchored to bobbins mounted on the copper bracket. The harnesses were then wrapped several times around the cryostat first stage and then mated to the D connectors. The harnesses were wrapped in 10 layers of MLI between copper bracket and at the first stage. The silicone diodes employed twisted shielded wire from the chamber feed through to the data aquistition rack. All grounds were properly wired to mitigate noise problems.

\section{TEST PROGRAM}

One of the objectives of this program was to determine the optimum fluid charge for proper loop operation with neon. The initial charge pressure of 280 psia was based on a similar calculation performed (Bugby, 1997) to arrive at a 40 grams of neon. Since the hot reservoir volume was somewhat larger than the initial test, 2550 versus $2250 \mathrm{cc}$, subsequent lowering of the charge pressure realized a lower saturation temperature, enabling the loop to operate in variable conductance mode. Tests were performed with fluid inventories between 1.93 and $1.58 \mathrm{MPa}$.

The following tests were conducted to characterize the loop performance under various fluid inventories: a) start up from a supercritical state, b) power cycle, c) sink temperature cycle, d) heat transport limit, e) low power operation for long duration, $\mathrm{f}$ ) reservoir temperature cycle. Details are presented in the following sections.

\section{Start Up}

The loop started reliably at each charge pressure, usually with two reservoir cycles. At a charge pressure of 1.58 $\mathrm{MPa}$, the loop was difficult to start and was not very stable. The minimum operating saturation pressure was near $0.69 \mathrm{MPa}$ for $1.93 \mathrm{MPa}$ charge level (corresponds to saturation temperature of $35.5 \mathrm{~K}$ ). Subsequent test conducted at 1.65 MPa charge level yielded minimum operating pressures around $0.34 \mathrm{MPa}$ (corresponds to saturation temperature of $32 \mathrm{~K}$ ). Figure 4 is a typical start up.

During start up, the cold reservoir and condenser are cooled below the critical point of neon (44.5K) at which point the fluid begins condensing rapidly. This is noted by the sharp decrease in system pressure. When the pressure begins to stabilize, a manual heater on the cold reservoir is activated at 9 watts for 20 to 30 seconds. This process shuttles cold liquid out of the reservoir and helps cool down the evaporator. A second reservoir cycle is normally needed before the temperature of the evaporator is below neon's critical temperature. A small amount of bias heat is maintained on the reservoir, 0.2 to 0.4 watts, which helps maintain positive control of the loop operating pressure. 0.5 watt is then applied to the evaporator to initiate fluid flow in the loop.

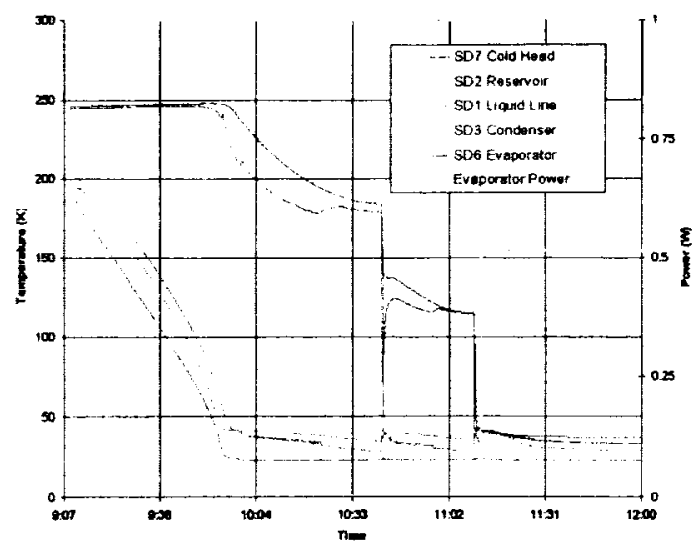

FIGURE 4. Start up, $1.93 \mathrm{MPa}$. 


\section{Low Power and Long Duration}

A low power, long duration test was performed to display the stability of the loop over a relatively long time period. Several long duration tests were conducted under various evaporator powers and reservoir control set points. Typical length for a long duration test would be 16 hours. The loop would be left unattended overnight and always ran stable until operator intervention for the next test.

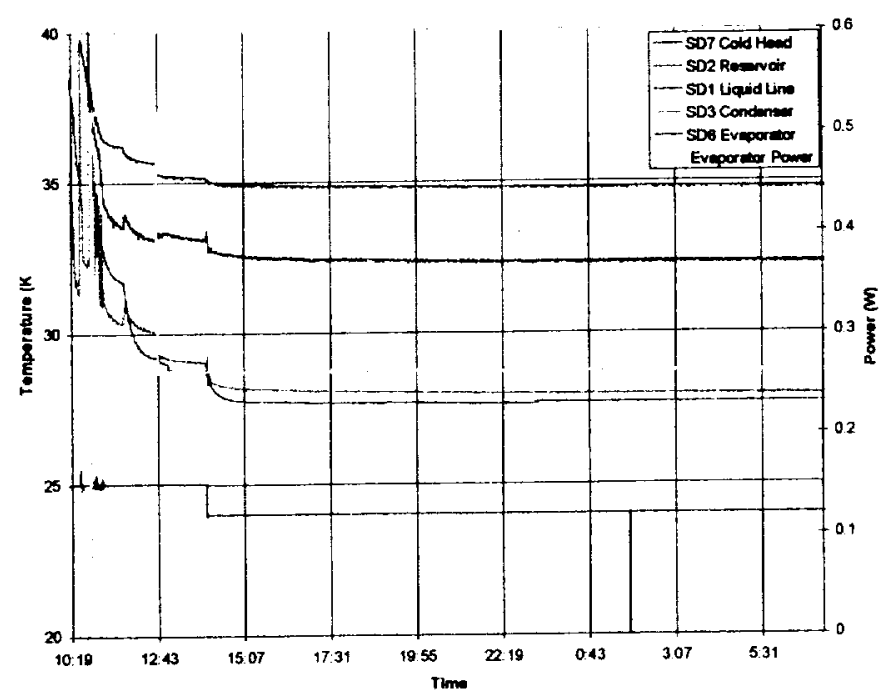

Figure 5. Low Power, Long Duration, 1.86 MPa.

The loop was very stable during this test but when the evaporator power was set to zero the evaporator dried out. This indicates that the parasitic heat load is not enough to run the loop. Figure 5 displays a low power ( 0.25 watt on evaporator) long duration test lasting roughly 16 hours.

\section{Power Cycle}

Several power cycle tests were performed to display the robustness of the loop during rapid changes in evaporator power. Typical power cycles included $0.5 / 2.0 / 0.5,0.25 / 2.25 / 0.25,0.5 / 1.5 / 0.5$. The condenser reacts with the evaporator as can be seen in figure 6. It was possible that the loop was running in a fixed conductance mode at 270 psia. It is also possible that with only one silicone diode on the condenser, the sensor did not read the true vapor temperature at higher powers.

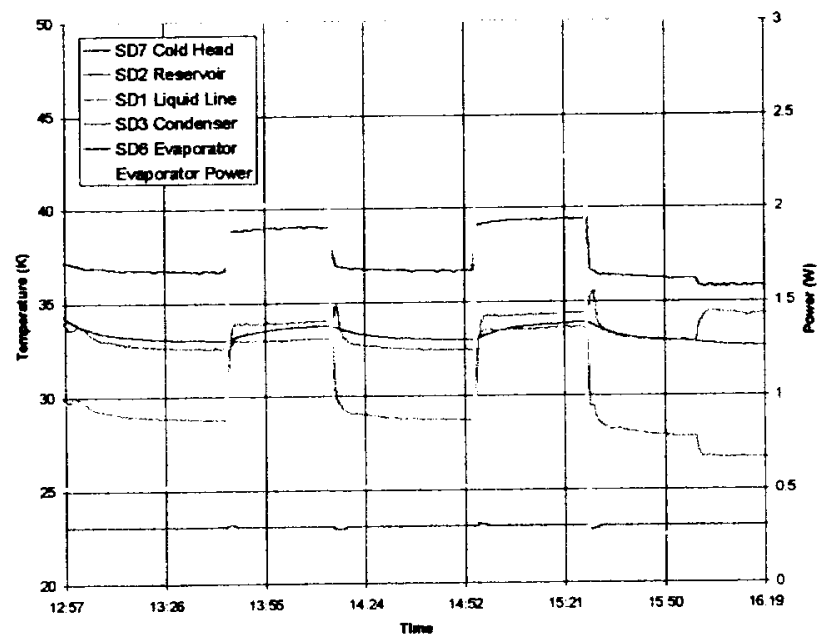

Figure 6. Power Cycle, $1.86 \mathrm{MPa}$. 


\section{Heat Transport Limit}

The heat transport limit of the loop was conducted by gradually increasing the heat load to the evaporator. The evaporator increases with an increasing heat load because a larger delta $T$ was needed in order to transfer the larger heat load. When the capillary limit was exceeded, however, the evaporator temperature would keep increasing without reaching a steady temperature. Figure 7 shows the loop reaching it's capillary limit at 2.75 watts. Subsequent tests consistently showed a heat transport limit of 2.75 watts. The loop could be quickly recovered if the power was lowered, as seen by the power cycle in figure 7 .

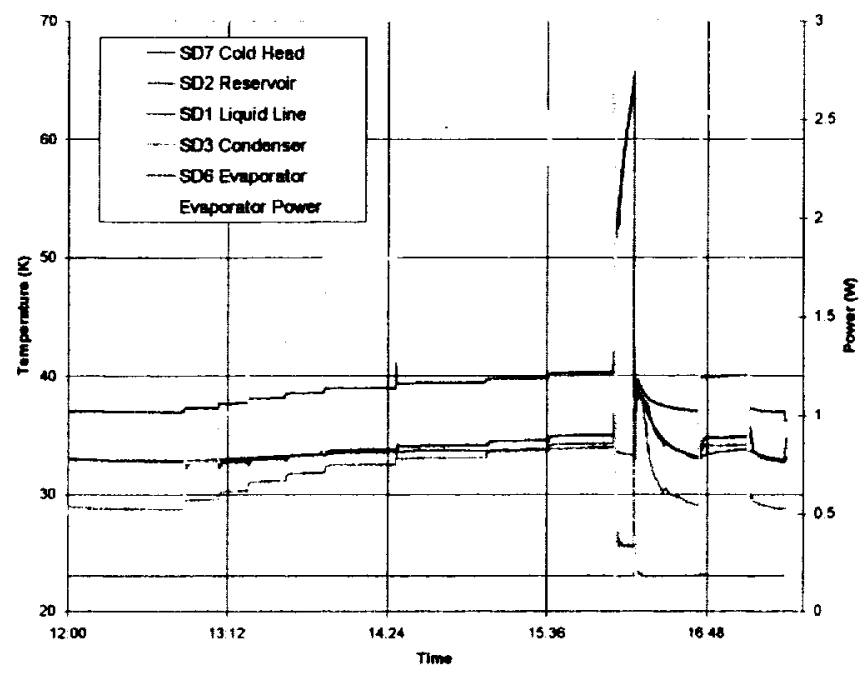

Figure 7. Heat Transport, Dryout and Recovory, $1.93 \mathrm{MPa}$.

\section{Condenser Cycling}

The condenser temperature was actively controlled though the use of a dedicated controller. This controller was extremely stable and gave us the flexibility to run various condenser temperature profiles. The testing was performed with and without reservoir control. Figure 8 shows a typical condenser sink cycle where the temperature was set to cycle from $25 \mathrm{~K}$ to $21 \mathrm{~K}$ back to $25 \mathrm{~K}$. The system responds as accordingly with no adverse affects on the system stability. No reservoir control was used for this test.

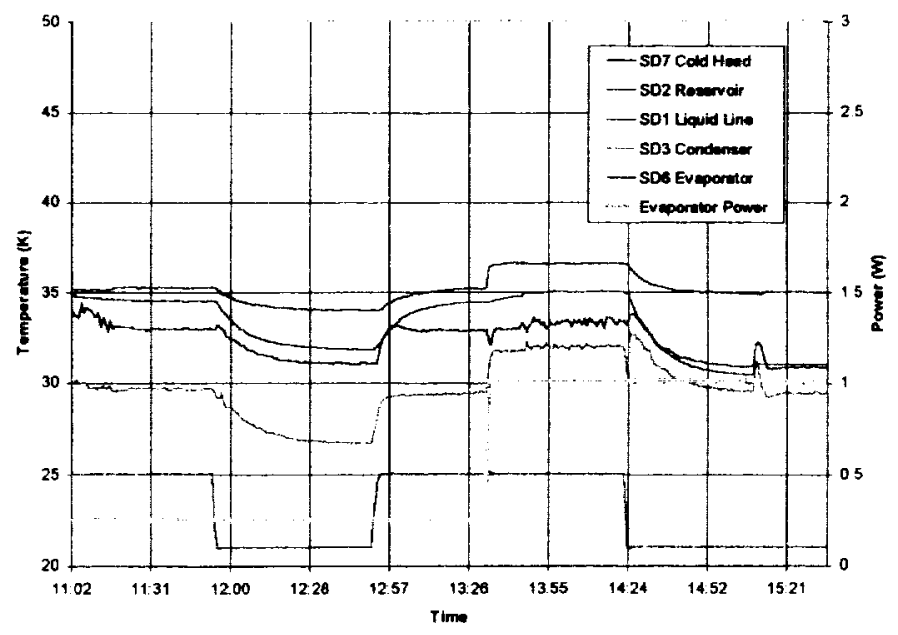

Figure 8. Condenser Cycling. 1.72 $\mathrm{MPa}$. 


\section{Reservoir Control}

The saturation temperature of the fluid in the cold reservoir governs the operating temperature of the loop. An absolute pressure transducer was used to verify the saturation temperature during this program. The control sensor which fed back to the temperature controller was located on the vapor phase of the reservoir as earlier test produced large temperature swings when the transducer was located in the liquid region. Once the parameters were adjusted on the PID temperature controller, the reservoir could be controlled very accurately to within 0.1 degree $\mathrm{K}$. This exhibits variable conductance operation when the heat load to the evaporator is varied while maintaining constant reservoir control temperature. Figure 9 displays this reservoir control during a power cycle and then a condenser cycle. The reservoir maintains control of the loop during both of these cycles. There is some change in the evaporator temperature due to the proximity of the temperature sensor to the heater.

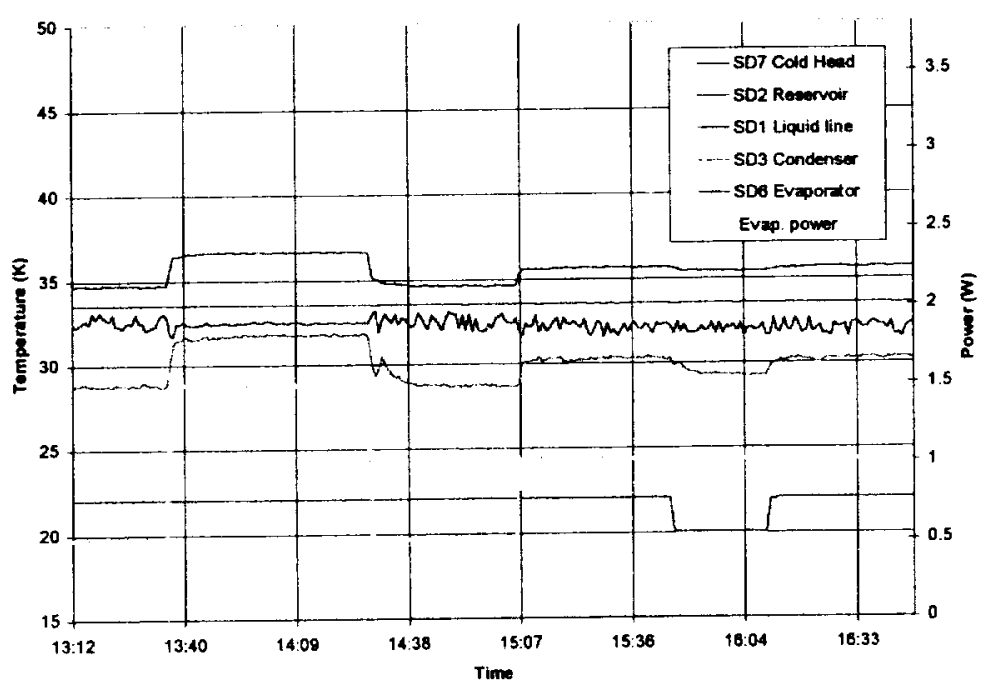

Figure 9. Reservoir Control during Power/Condenser Cycles, 1.65MPa.

\section{CONCLUSIONS}

The operation of a neon cryogenic capillary pumped loop is readily achievable and exhibits robust operation under various operating conditions. The loop started successfully with one or two reservoir cycles and adapted to rapid changes of heat load and sink temperature. The loop operated stable at $0.25 \mathrm{w}$ and 2.25 watt.

\section{ACKNOWLWEDGMENTS}

We would like to thanks the NGST project at NASA Goddard Space Flight Center for sponsoring this work.

\section{REFERENCES}

Baumann, J. et al., "Development of the Cryogenic Capillary Pumped Loop", IECEC-98-I137, presented at $33^{\text {rd }}$ Intersociety Engineering Conference on Energy Conversion, Colorado Springs, CO, August 2-6, 1998.

Bugby, D. C. et al., "Experimental Investigation of a Neon Cryogenic Capillary Pumped Loop", IECEC - 97-I272, presented at $32^{\text {nd }}$ Intersociety Engineering Conference on Energy Conversion, Honolulu, HA, July $27^{\text {th }}-$ August $1^{\text {st }}, 1997$.

Ku, J., et al., "Flight Testing of a Cryogenic Capillary Pumped Loop", IECEC - 99 - 042 presented at $34^{\text {th }}$ Intersociety

Engineering Conference on Energy Conversion, Vancouver, British Columbia, August 1-5, 1999. 
